\title{
Soft gluons and top quark pair production
}

\author{
Li Lin Yang ${ }^{* \dagger}$ \\ School of Physics and State Key Laboratory of Nuclear Physics and Technology, Peking \\ University, Beijing 100871, China \\ Collaborative Innovation Center of Quantum Matter, Beijing, China \\ Center for High Energy Physics, Peking University, Beijing 100871, China \\ E-mail: yanglilinepku.edu.cn
}

\begin{abstract}
In this talk we summarize two recent efforts towards improving the theoretical predictions for the differential cross sections in top quark pair production. Both are related to the effect of soft gluons. The first result is the analytic calculation of the NNLO massive soft function which is an important ingredient in the resummation of threshold logarithms. The second is the matched NNLO+NNLL' predictions which combine state-of-the-art NNLO QCD calculations with double resummation at NNLL' accuracy of threshold logarithms arising from soft gluon emissions and of small mass logarithms.
\end{abstract}

Loops and Legs in Quantum Field Theory (LL2018)

29 April 2018 - 04 May 2018

St. Goar, Germany

\footnotetext{
* Speaker.

${ }^{\dagger}$ Based on collaborations with M. Czakon, A. Ferroglia, D. Heymes, A. Mitov, B. D. Pecjak, D. J. Scott, G. Wang, X. Wang, X. Xu and H. X. Zhu. 


\section{Introduction}

Top quark pair production is one of the most important processes at the Large Hadron Collider (LHC). It allows us to precisely study the properties of the top quark which are related to many important questions in particle physics, such as the hierarchy problem, the stability of the electroweak vacuum, as well as the origin of fermion masses. Top quark pair production is also a major background to the searches for many rare processes in the standard model (SM) and in new physics models beyond the SM.

Currently, the best fixed-order calculation in quantum chromodynamics (QCD) for top quark pair production is at the next-to-next-to-leading order (NNLO) [1-7]. Despite the high precision of the NNLO result, the complicated kinematics of $t \bar{t}$ production makes it necessary to consider even higher order corrections. This is particularly important since the large collider energy of the LHC enables the study of "boosted" top quark pairs, where the energies of the top quarks are much larger than their rest mass $m_{t}$. In [7], it has been found that the NNLO QCD differential cross sections in the boosted regime are rather sensitive to the choice of factorization and renormalization scales. This scale dependence can be dramatically reduced by resumming certain towers of large logarithms to all orders in the strong coupling $\alpha_{s}[8,9]$. These include not only the threshold logarithms which arise when the partonic center-of-mass energy approaches the $t \bar{t}$ invariant mass $M$, but also the small-mass logarithms of the form $\ln ^{n}\left(m_{t}^{2} / M^{2}\right)$ which develop in the boosted region $M \gg m_{t}$.

The resummation of the large logarithms starts from the factorization formula for the differential cross section with respect to the $t \bar{t}$ invariant mass $M$ and the scattering angle $\theta$. It can be conveniently written in the moment space after a Mellin transform as

$$
\frac{d^{2} \widetilde{\sigma}(N)}{d M d \cos \theta}=\frac{8 \pi \beta}{3 s M} \sum_{i j} \widetilde{\mathscr{L}}_{i j}\left(N, \mu_{f}\right) \widetilde{c}_{i j}\left(N, M, \beta, \cos \theta, \mu_{f}\right),
$$

where $N$ is the Mellin moment, $\mu_{f}$ is the factorization scale, $\beta=\sqrt{1-4 m_{t}^{2} / M^{2}}, \widetilde{\mathscr{L}}_{i j}$ is the parton luminosity function, and $\widetilde{c}_{i j}$ is the hard-scattering kernel. The threshold limit corresponds to $N \rightarrow$ $\infty$, where the hard-scattering kernel develops large logarithms of the form $\ln ^{n} N$. In order to resum these threshold logarithms, one exploits the factorization formula [10-12]

$$
\begin{aligned}
\widetilde{c}_{i j}(N, M, \beta, \cos \theta, \mu)=\operatorname{Tr}\left[\boldsymbol{H}_{i j}^{m}\left(\ln \frac{M^{2}}{\mu^{2}}, \beta, \cos \theta, \mu\right)\right. \\
\left.\quad \times \widetilde{\boldsymbol{s}}_{i j}^{m}\left(\ln \frac{M^{2}}{\bar{N}^{2} \mu^{2}}, \beta, \cos \theta, \mu\right)\right]+\mathscr{O}\left(\frac{1}{N}\right),
\end{aligned}
$$

where $\bar{N}=N e^{\gamma_{E}}$ with $\gamma_{E}$ the Euler constant, while $\boldsymbol{H}_{i j}^{m}$ and $\widetilde{\boldsymbol{s}}_{i j}^{m}$ are the massive hard and soft functions, which are both matrices in the color space as indicated by the bold symbols. The resummation then proceeds by choosing an appropriate hard scale $\mu_{h}$ for $\boldsymbol{H}_{i j}^{m}$ and an appropriate soft scale $\mu_{s}$ for $\widetilde{\boldsymbol{s}}_{i j}^{m}$, and evolving the two functions to the factorization scale $\mu_{f}$ via their renormalization group equations (RGEs).

The factorization formula (1.2) is valid whether or not the top quarks are boosted. However, in the boosted limit $M \gg m_{t}$ or $\beta \rightarrow 1$, the massive hard and soft functions $\boldsymbol{H}_{i j}^{m}$ and $\widetilde{\boldsymbol{s}}_{i j}^{m}$ themselves 
develop large logarithms of the form $\ln ^{n}\left(m_{t}^{2} / M^{2}\right)$ which also requires resummation. In [13], it was shown that the massive hard and soft functions can be further factorized in the boosted limit as

$$
\begin{aligned}
\boldsymbol{H}_{i j}^{m}\left(\ln \frac{M^{2}}{\mu^{2}}, \beta, \cos \theta, \mu\right) & =\boldsymbol{H}_{i j}\left(\ln \frac{M^{2}}{\mu^{2}}, \cos \theta, \mu\right) C_{D}^{2}\left(\ln \frac{m_{t}^{2}}{\mu^{2}}, \mu\right)+\mathscr{O}\left(\frac{m_{t}^{2}}{M^{2}}\right), \\
\widetilde{\boldsymbol{s}}_{i j}^{m}\left(\ln \frac{M^{2}}{\bar{N}^{2} \mu^{2}}, \beta, \cos \theta, \mu\right) & =\widetilde{\boldsymbol{s}}_{i j}\left(\ln \frac{M^{2}}{\bar{N}^{2} \mu^{2}}, \cos \theta, \mu\right) \tilde{s}_{D}^{2}\left(\ln \frac{m_{t}^{2}}{\bar{N}^{2} \mu^{2}}, \mu\right)+\mathscr{O}\left(\frac{m_{t}^{2}}{M^{2}}\right),
\end{aligned}
$$

where $\boldsymbol{H}_{i j}$ and $\widetilde{\boldsymbol{s}}_{i j}$ are massless hard and soft functions describing the production of a highly boosted top quark pair, while $C_{D}$ and $\tilde{s}_{D}$ describe the fragmentation of a nearly massless top quark. Using this double factorization, one can simultaneously resum the threshold logarithms and the smallmass logarithms in the boosted region via the RGEs.

In order to achieve the resummation at the next-to-next-to-leading logarithmic (NNLL) accuracy, we need to know the various functions in the factorization formulas (1.2) and (1.3) to the next-to-leading order (NLO), and the anomalous dimensions governing their evolution to order $\alpha_{s}^{2}$. These ingredients for the un-boosted case have been collected in $[12,14,15]$ and the NNLL resummation of the threshold logarithms was performed in [12]. This result will be denoted as NNLL in the following, where the subscript "m" means "massive". In the boosted case, it is possible to improve the resummation accuracy to NNLL' by including the NNLO contributions to the functions $\boldsymbol{H}_{i j}, \widetilde{\boldsymbol{s}}_{i j}, C_{D}$ and $\tilde{s}_{D}[13,16]$. Among them the NNLO massless soft function was calculated in [17]. This NNLL ' ("b" meaning "boosted") resummation can be combined with the NNLL $L_{m}$ resummation to obtain an $\mathrm{NNLL}_{\mathrm{b}+\mathrm{m}}^{\prime}$ result valid both in the un-boosted and boosted regions. This was further matched to the NLO fixed-order calculation and finally arrived at the NLO+NNLL' result in [8].

In this talk, we present two recent efforts towards improving the NLO+NNLL' predictions. In Section 2, we discuss the calculation of the NNLO massive soft function which can be used in the future to improve the resummation accuracy of the threshold logarithms to $\mathrm{NNLL}_{\mathrm{m}}^{\prime}$. In Section 3, we discuss the construction of the NNLO+NNLL' predictions combining the NNLO calculation with the double resummation at the $\mathrm{NNLL}_{\mathrm{b}+\mathrm{m}}^{\prime}$ precision. We summarize in Section 4.

\section{The NNLO massive soft function}

The massive soft function is an important ingredient in the factorization formula (1.2) which is key to the resummation of threshold logarithms. In order to perform the resummation at the NNLL' accuracy, it is necessary to calculate the soft function to the NNLO. This has been accomplished in [18]. In this section, we briefly review the main results of that work.

The soft function describes the interactions of soft gluons with the hard partons involved in a scattering process. It is defined in terms of the soft Wilson lines

$$
\boldsymbol{S}_{i}(x)=\mathscr{P} \exp \left(i g_{s} \int_{-\infty}^{0} d s v_{i} \cdot A^{a}\left(x+s v_{i}\right) \boldsymbol{T}_{i}^{a}\right)
$$

where $\mathscr{P}$ denotes path ordering, $v_{i}$ is a (incoming) 4 -vector parallel to the momentum of the $i$-th hard parton, which satisfies $v_{i}^{2}=0$ for massless partons and $v_{i}^{2}>0$ for massive ones. The boldface 
$\boldsymbol{T}_{i}^{a}$ is the color generator associated with the $i$-th hard parton in the color-space formalism $[19,20]$. Specifically, we consider the $t \bar{t}$ production process

$$
h_{1}\left(p_{1}\right)+h_{2}\left(p_{2}\right) \rightarrow t\left(p_{3}\right)+\bar{t}\left(p_{4}\right)+X_{s}\left(p_{s}\right)
$$

where $h_{1}$ and $h_{2}$ are two incoming massless partons, and $X_{s}$ represents additional soft radiations which are described by the soft function. We work in the limit $p_{s} \rightarrow 0$, where $M^{2}=\left(p_{1}+p_{2}\right)^{2}=$ $\left(p_{3}+p_{4}\right)^{2}$, and the scattering angle $\theta$ is defined as the angle between $\vec{p}_{3}$ and $\vec{p}_{1}$ in the partonic center-of-mass frame. The bare momentum-space soft function is defined by

$$
\boldsymbol{S}_{\mathrm{bare}}(\omega, \beta, \cos \theta) \equiv \frac{1}{d_{R}} \sum_{X_{s}}\left\langle 0\left|\prod_{i=1}^{4} \boldsymbol{S}_{i}^{\dagger}(0)\right| X_{s}\right\rangle\left\langle X_{S}\left|\prod_{i=1}^{4} \boldsymbol{S}_{i}(0)\right| 0\right\rangle \delta\left(\omega-v_{0} \cdot p_{X_{s}}\right),
$$

where the reference vector $v_{0}=(2,0,0,0)$, and $\omega$ represents ( 2 times) the energy of the additional soft partons. We have included a normalization factor $d_{R}=3$ for $h_{1} h_{2}=q \bar{q}$ and $d_{R}=8$ for $h_{1} h_{2}=$ gg. It is convenient to perform a Mellin or Laplace transform into the moment space

$$
\tilde{\boldsymbol{s}}_{\text {bare }}(\Lambda, \beta, \cos \theta)=\int_{0}^{\infty} d \omega \exp \left(-\frac{\omega}{\Lambda e^{\gamma_{E}}}\right) \boldsymbol{S}_{\text {bare }}(\omega, \beta, \cos \theta),
$$

where $\Lambda$ is a soft momentum scale in the moment space which will be identified with $M / \bar{N}$ later.

The next-to-leading order (NLO) contribution to the bare soft function has been calculated in [12], which is rather generic and can be applied to many processes besides $t \bar{t}$ production, e.g., the $t \bar{t} H$ production process [21,22]. In order to calculate the NNLO soft function, it is necessary to have the NLO term to higher orders in the dimensional regulator $\varepsilon=(4-d) / 2$, where $d$ is the space-time dimension. This can be done using the integration-by-parts (IBP) identities $[23,24]$ and the method of differential equations [25-27]. The results can be obtained by solving the differential equations and are expressed in terms of generalized polylogarithms (GPLs) [28] to all orders in $\varepsilon$.

The calculation of the bare NNLO contribution is more involved, but can proceed with similar techniques. One subtlety in the calculation is the boundary conditions for the differential equations. We choose the boundary to be $\beta \rightarrow 0$. However, some of the virtual-real integrals develop Coulomb-type singularities in this limit. To illustrate this, we consider the integral family defined by

$$
\begin{array}{r}
F_{a_{1}, a_{2}, a_{3}, a_{4}, a_{5}, a_{6}, a_{7}, a_{8}}^{(4)} \equiv \int d^{d} k d^{d} l \delta\left(\omega-v_{0} \cdot k\right) \operatorname{Disc}\left[\left(k^{2}\right)^{-a_{1}}\right]\left[l^{2}\right]^{-a_{2}}\left[(k+l)^{2}\right]^{-a_{3}}\left[v_{1} \cdot k\right]^{-a_{4}} \\
\times\left[v_{1} \cdot l\right]^{-a_{5}}\left[v_{4} \cdot(k+l)\right]^{-a_{6}}\left[v_{3} \cdot k\right]^{-a_{7}}\left[v_{3} \cdot l\right]^{-a_{8}}
\end{array}
$$

where

$$
\operatorname{Disc}\left[\left(k^{2}\right)^{-a_{1}}\right] \equiv \frac{1}{2 \pi i}\left[\left(k^{2}+i 0\right)^{-a_{1}}-\left(k^{2}-i 0\right)^{-a_{1}}\right] .
$$

This integral family has 21 master integrals which satisfy differential equations

$\partial_{\beta} \vec{g}^{(4)}(\varepsilon, \beta, \cos \theta)=\varepsilon\left(-\frac{\hat{a}^{(4)}}{\beta-1}+\frac{\hat{b}^{(4)}}{\beta}+\frac{\hat{c}^{(4)}}{\beta+1}-\frac{\hat{d}^{(4)}}{\beta-1 / \cos \theta}+\frac{\hat{e}^{(4)}}{\beta+1 / \cos \theta}\right) \vec{g}^{(4)}(\varepsilon, \beta, \cos \theta)$, 
where $\hat{a}^{(4)}, \hat{b}^{(4)}, \hat{c}^{(4)}, \hat{d}^{(4)}$ and $\hat{e}^{(4)}$ are $21 \times 21$ matrices depending only on $\cos \theta$. All but two of the master integrals are regular in the limit $\beta \rightarrow 0$ and can be readily evaluated at the boundary. The remaining two are logarithmic divergent as $\beta \rightarrow 0$, and we need to extract their asymptotic behavior near the boundary. As an example, we have

$$
\begin{aligned}
g_{9}^{(4)}(\varepsilon, \beta, \cos \theta) & =-\frac{i e^{-2 i \pi \varepsilon} \Gamma(1-2 \varepsilon) \omega^{4 \varepsilon} \beta}{4 \pi^{3-2 \varepsilon} \Gamma^{2}(-\varepsilon) \Gamma(2 \varepsilon)} \int \frac{d^{d} k d^{d} l \delta^{+}\left(k^{2}\right) \delta\left(\omega-v_{0} \cdot k\right)}{\left(l^{2}+i 0\right)\left(-v_{1} \cdot k\right)\left(v_{3} \cdot l+i 0\right)\left[-v_{4} \cdot(k+l)+i 0\right]} \\
& \stackrel{\beta \rightarrow 0}{\approx} \frac{\left(e^{-2 i \pi \varepsilon}-1\right) \beta^{2 \varepsilon} \Gamma(1-2 \varepsilon) \Gamma(1+\varepsilon)}{2^{3-4 \varepsilon} \Gamma(1-\varepsilon)}
\end{aligned}
$$

Given the boundary conditions, the bare NNLO soft function can be solved from the differential equations in terms of GPLs.

The bare soft function contains ultraviolet divergences and requires renormalization in the form

$$
\tilde{\boldsymbol{s}}(L, \beta, \cos \theta, \mu)=\lim _{\varepsilon \rightarrow 0} \boldsymbol{Z}_{s}^{\dagger}(L, \beta, \cos \theta, \mu) \tilde{\boldsymbol{s}}_{\text {bare }}(\Lambda, \beta, \cos \theta) \boldsymbol{Z}_{s}(L, \beta, \cos \theta, \mu)
$$

where $L \equiv \ln \left(\Lambda^{2} / \mu^{2}\right)$, and the renormalization matrix $\boldsymbol{Z}_{s}$ can be constructed from the anomalous dimension matrix $\Gamma_{s}(L, \beta, \cos \theta, \mu)$ obtained in [14,15,29]. It is interesting to note that the 3-parton correlated divergences arising in our calculation are exactly cancelled by the corresponding term in the anomalous dimension matrix, which is a highly non-trivial check of our result. We have also verified the $\beta \rightarrow 0$ limit of the renormalized soft function against the known results in $[30,31]$ and find complete agreements. A more interesting and non-trivial limit is $\beta \rightarrow 1$, which corresponds to highly boosted top quarks. In this limit the massive soft function can be further factorized as in Eq. (1.3). We have checked that our result indeed satisfies that factorization formula, and this allows us to extract the NNLO expression of the soft fragmentation function $\tilde{s}_{D}$.

\section{Resummation at NNLO+NNLL' in QCD}

In [8], the $\mathrm{NNLL}_{\mathrm{b}+\mathrm{m}}^{\prime}$ resummed result is only matched to the NLO fixed-order result. With the availability of the NNLO result with dynamic renormalization and factorization scales [7], it is desirable to combined these two state-of-the-art calculations, which was finally achieved in [9]. This is the first time a resummed calculation at full NNLO+NNLL' accuracy in QCD for a process with non-trivial color structure has been completed at the differential level.

Technically, the NNLO+NNLL' result involves three different contributions, two of which contains all-order information. Therefore we need to combine them very carefully to ensure that there is no double-counting (or triple-counting) at any order in $\alpha_{s}$. We first match the resummation formulas in the soft and boosted-soft limit with each other. To do so, we need to remove the overlap between the $\mathrm{NNLL}_{b}^{\prime}$ and $\mathrm{NNLL}_{m}$ results to all orders in $\alpha_{s}$. This can be done by exploiting the fact that the boosted-soft resummation formula is the small-mass limit of the soft-gluon resummation formula at any given order in $\alpha_{s}$. The combined $\mathrm{NNLL}_{\mathrm{b}+\mathrm{m}}^{\prime}$ result is thus given by

$$
d \sigma^{\mathrm{NNLL}} \mathrm{b}_{\mathrm{b}+\mathrm{m}}^{\prime}=d \sigma^{\mathrm{NNLL}}+\left(d \sigma^{\mathrm{NNLL}_{\mathrm{m}}}-\left.d \sigma^{\mathrm{NNLL}_{\mathrm{m}}}\right|_{m_{t} \rightarrow 0}\right),
$$


where the terms in the parenthesis account for contributions which are suppressed by $m_{t} / M$ in the boosted-soft limit and thus not included in the $\mathrm{NNLL}_{b}^{\prime}$ result. Matching with the NNLO calculation then proceed by subtracting the NNLO expansion of the resummed formula

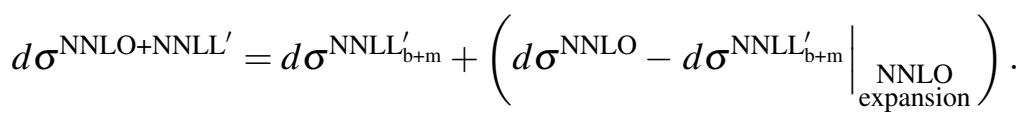

With the above formulas, it is straightforward to perform the matching and obtain the NNLO+NNLL' predictions for the $t \bar{t}$ invariant mass distribution as well as the top quark transverse momentum distribution. However, due to the complicated kinematics of $t \bar{t}$ production, one should be careful about the choice of the factorization scale as well as the matching scales for each of the functions in the factorization formula (1.2) and (1.3). In [7], it has been found that the $t \bar{t}$ invariant mass distribution are quite sensitive to the choice of the factorization scale in the boosted region, even at NNLO. By studying the convergence of the perturbative series, it was argued that the optimal choice should be

$$
\mu_{f}=\frac{H_{T}}{4} \equiv \frac{1}{4}\left[\sqrt{p_{T, t}^{2}+m_{t}^{2}}+\sqrt{p_{T, \bar{t}}^{2}+m_{t}^{2}}\right],
$$

instead of correlating with $M$. This fact also has implications for the choices of the other matching scales in the resummation formula, especially the hard scale $\mu_{h}$ and the soft scale $\mu_{s}$. In [8], the hard scale was chosen to be correlated with $M$. However, a closer look at the hard function in the $g g$-channel reveals that in the boosted limit, the $t$ - and $u$-channel propagators enhance the forward and backward regions:

$$
\begin{aligned}
& m_{t}^{2}-\left.\left(p_{1}-p_{3}\right)^{2}\right|_{m_{t} \rightarrow 0} \approx \frac{M^{2}}{2}(1-\cos \theta)+m_{t}^{2} \cos \theta \stackrel{\cos \theta \rightarrow 1}{\longrightarrow} p_{T, t}^{2}+m_{t}^{2} \approx H_{T}^{2} / 4, \\
& m_{t}^{2}-\left.\left(p_{2}-p_{3}\right)^{2}\right|_{m_{t} \rightarrow 0} \approx \frac{M^{2}}{2}(1+\cos \theta)-m_{t}^{2} \cos \theta \stackrel{\cos \theta \rightarrow-1}{\longrightarrow} H_{T}^{2} / 4 .
\end{aligned}
$$

As a result, the hard function is sensitive to the scale $H_{T} / 2$ instead of $M$ when the top quarks are highly boosted. The analytic form and the numeric behavior of the hard function in the boosted region then lead to the default choice $\mu_{h}=H_{T} / 2$, as concluded in [9]. The choice of the soft scale, on the other hand, is not as obvious. By studying the perturbative convergence of the massless soft function, [9] has identified the default choice $\mu_{s}=H_{T} / \bar{N}$. This choice is also supported by the behavior of the massive soft function introduced in the last section [18]. In the following, we will adopt these default choices, together with the choice of $\mu_{f}$ as in Eq. (3.3).

We now show some phenomenological results at the LHC with $\sqrt{s}=13 \mathrm{TeV}$. We use the NNPDF3.0 NNLO PDF sets with $\alpha_{s}\left(M_{Z}\right)=0.118$ [32], and take $m_{t}=173.3 \mathrm{GeV}$. The perturbative uncertainties are estimated by varying the various scales separately and adding the resulting uncertainties in quadrature. In Fig. 1, we show the results for the absolute (left plot) and the normalized (right plot) $t \bar{t}$ invariant mass distribution. A remarkable feature of this figure is that the NNLO+NNLL' and NNLO results are in close agreement in the whole range of $M$ when $\mu_{f}=H_{T} / 4$ is chosen. To add context to this result, we display in Fig. 2 the results for the cross section in a sample bin $M=[2500-3000] \mathrm{GeV}$ in the boosted region. This figure deliver a couple of important messages. Firstly, the NNLO+NNLL' result is rather stable against switching the factorization scale between $H_{T}$-based and $M$-based schemes. This implies that the even higher order corrections 

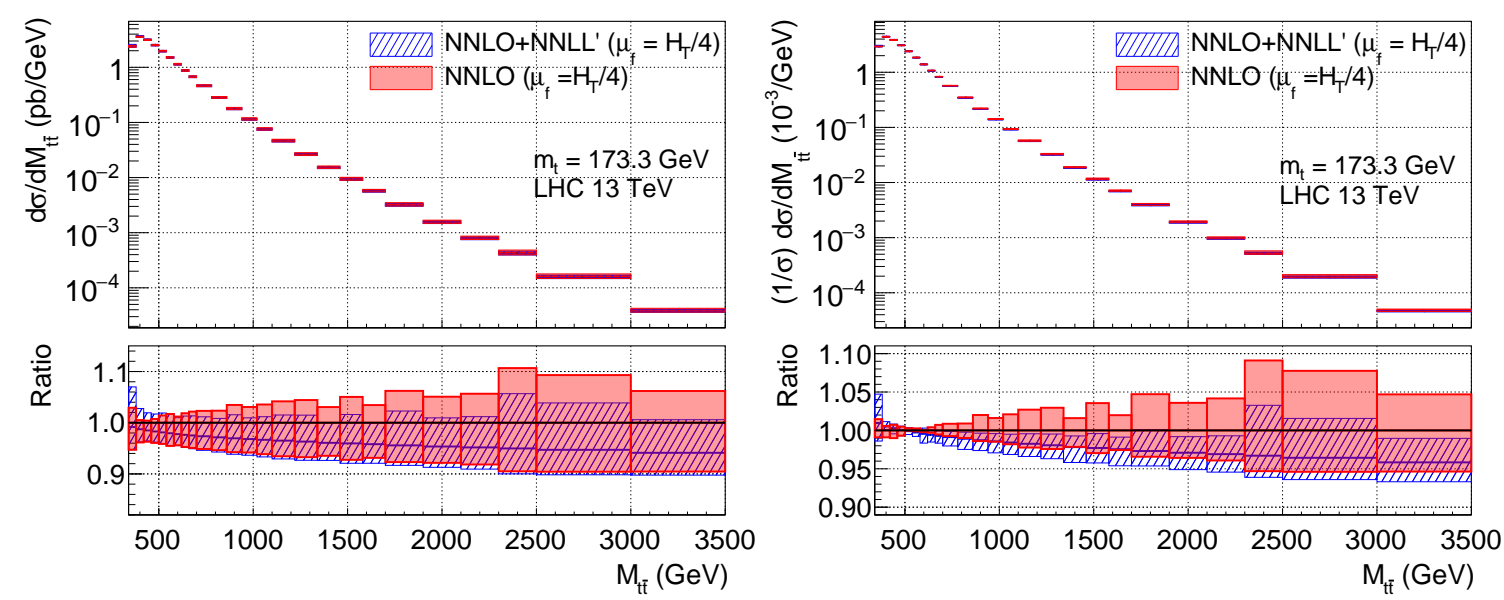

Figure 1: Results for the absolute (left) and normalized (right) top-pair invariant mass distribution at the LHC with $\sqrt{s}=13 \mathrm{TeV}$. In all cases the ratio is to the NNLO result with $\mu_{f}=H_{T} / 4$. The uncertainty bands reflect scale variations.

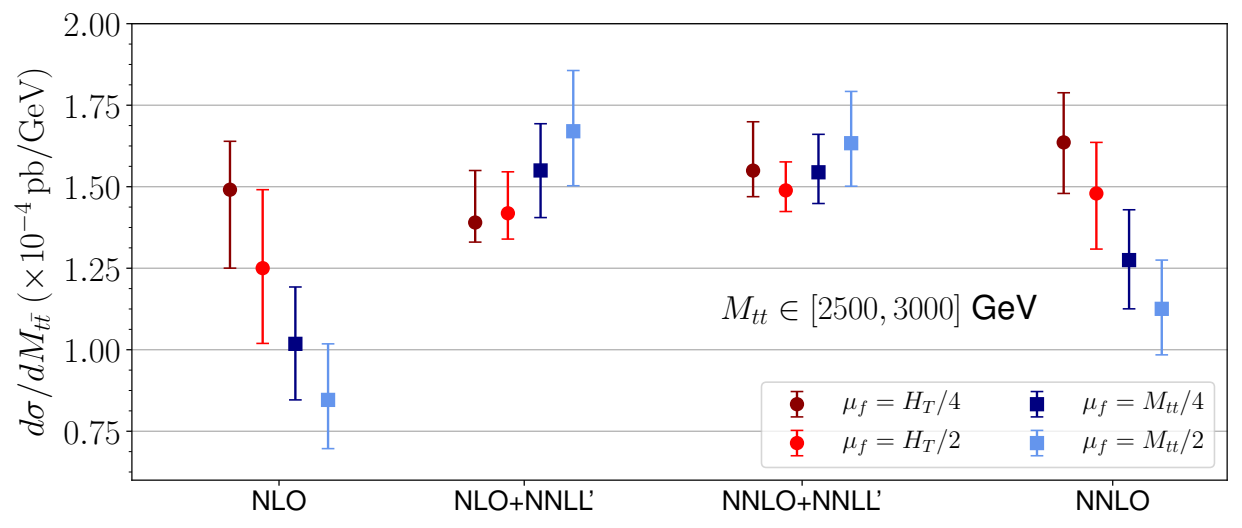

Figure 2: Cross sections obtained in a sample bin $M=[2500-3000] \mathrm{GeV}$ in the boosted region. The default value of $\mu_{f}$ is indicated explicitly, and the error bars represent perturbative uncertainties estimated through scale variations.

to the NNLO+NNLL' result are not so important. On the other hand, the NNLO result changes drastically when switching the schemes. In particular, higher order contributions beyond NNLO encoded in the resummation produce a very large effect for the choice $\mu_{f}=M / 2$, as already forseen in [8]. Given these observations, the close compatibility between the NNLO+NNLL' result (with either scale choice) and the NNLO result with $\mu_{f}=H_{T} / 4$ is a highly non-trivial fact. This provides an important confirmation of the result of [7], which favors the choice $\mu_{f}=H_{T} / 4$ for the fixed-order calculation of the $t \bar{t}$ invariant mass distribution. The overall picture emerging from the above analysis is that the perturbative description of the top-quark pair invariant mass distribution is under good control.

Results for the absolute (normalized) average top/anti-top $\left(p_{T, \text { avt }}\right)$ distribution at NNLO and NNLO+NNLL' are shown in the left (right) panel of figure 3. The NNLO results (with which 

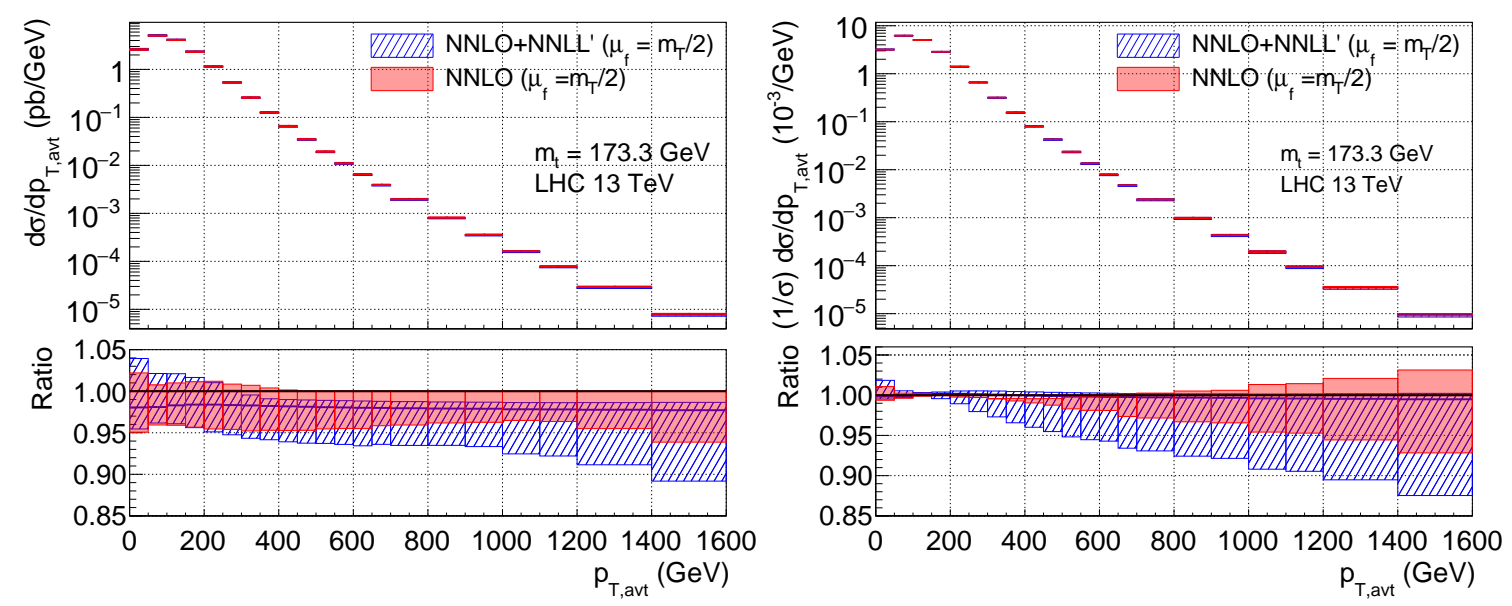

Figure 3: Results for the absolute (left) and normalized (right) $p_{T, \text { avt }}$ distributions at the LHC with $\sqrt{s}=$ $13 \mathrm{TeV}$. In all cases the ratio is to the NNLO result with $\mu_{f}=m_{T} / 2$.

resummation is matched) have been calculated using the definition

$$
\frac{d \sigma}{d p_{T, \mathrm{avt}}}=\frac{1}{2}\left(\frac{d \sigma}{d p_{T, t}}+\frac{d \sigma}{d p_{T, \bar{t}}}\right)
$$

The default factorization scale is chosen to be $\mu_{f}=m_{T} / 2$ (where $m_{T}$ refers to the transverse mass of either the top or anti-top quark depending on the distribution under consideration), which is favored by the study of [7]. We see that the NNLO+NNLL' result is consistent with the NNLO one. On the other hand, it has been found that upgrading matching with fixed-order from NLO+NNLL' to NNLO+NNLL' is an important effect for the $p_{T}$ distributions, especially in reducing the scale uncertainties in the high $p_{T}$ region. This is an important fact to keep in mind when using NLObased Monte Carlo event generators to model $p_{T}$ distributions.

\section{Summary}

To summarize, in this talk we have introduced two recent efforts towards improving the theoretical predictions for the differential cross sections in top quark pair production. Both are related to the effect of soft gluons.

The first result is the analytic calculation of the NNLO massive soft function which is an important ingredient in the resummation formula of threshold logarithms [18]. We find that it can be entirely written in terms of GPLs, which makes it efficient for numerical evaluation. Our result represents the first ever NNLO soft function for processes involving a non-trivial color structure and two massive partons with full velocity dependence. We show that in the boosted limit, the massive soft function exhibits the expect factorization property of mass logarithms, which leads to a consistent extraction of the soft fragmentation function. We also find full agreement with the NNLO massless soft function in [17], up to the three-parton virtual-real contributions not calculated in that paper. 
The second result is the matched NNLO+NNLL' predictions which combine state-of-the-art NNLO QCD calculations with double resummation at NNLL' accuracy of threshold logarithms arising from soft gluon emissions and of small mass logarithms [9]. This is the first time a resummed calculation at full NNLO+NNLL' accuracy in QCD for a process with non-trivial color structure has been completed at the differential level. Of main interest to us is the stability of the $t \bar{t}$ invariant mass and top-quark $p_{T}$ distributions in the boosted regime where fixed order calculations may become strongly dependent on the choice of dynamic scales. With the help of numeric and analytic arguments we confirm that the choice for the factorization and renormalization scales advocated in [7] is indeed optimal. We further derive a set of optimized kinematics-dependent scales for the matching functions which appear in the resummed calculations. Our NNLO+NNLL' prediction for the top-pair invariant mass is significantly less sensitive to the choice of factorization scale than the fixed order prediction, even at NNLO. Notably, the resummed and fixed order calculations are in nearly perfect agreement with each other in the full $M$ range when the optimal dynamic scale is used. For the top-quark $p_{T}$ distribution the resummation performed here has less of an impact and instead we find that upgrading the matching with fixed-order from NLO+NNLL' to NNLO+NNLL' to be an important effect, a point to be kept in mind when using NLO-based Monte Carlo event generators to calculate this distribution.

\section{References}

[1] P. Bärnreuther, M. Czakon and A. Mitov, Phys. Rev. Lett. 109, 132001 (2012) [arXiv:1204.5201 [hep-ph]].

[2] M. Czakon and A. Mitov, JHEP 1212, 054 (2012) [arXiv:1207.0236 [hep-ph]].

[3] M. Czakon and A. Mitov, JHEP 1301, 080 (2013) [arXiv:1210.6832 [hep-ph]].

[4] M. Czakon, P. Fiedler and A. Mitov, Phys. Rev. Lett. 110, 252004 (2013) [arXiv:1303.6254 [hep-ph]].

[5] M. Czakon, P. Fiedler and A. Mitov, Phys. Rev. Lett. 115, no. 5, 052001 (2015) [arXiv:1411.3007 [hep-ph]].

[6] M. Czakon, D. Heymes and A. Mitov, Phys. Rev. Lett. 116, no. 8, 082003 (2016) [arXiv:1511.00549 [hep-ph]].

[7] M. Czakon, D. Heymes and A. Mitov, JHEP 1704, 071 (2017) [arXiv:1606.03350 [hep-ph]].

[8] B. D. Pecjak, D. J. Scott, X. Wang and L. L. Yang, Phys. Rev. Lett. 116, no. 20, 202001 (2016) [arXiv:1601.07020 [hep-ph]].

[9] M. Czakon, A. Ferroglia, D. Heymes, A. Mitov, B. D. Pecjak, D. J. Scott, X. Wang and L. L. Yang, JHEP 1805, 149 (2018) [arXiv:1803.07623 [hep-ph]].

[10] N. Kidonakis and G. F. Sterman, Phys. Lett. B 387, 867 (1996).

[11] N. Kidonakis and G. F. Sterman, Nucl. Phys. B 505, 321 (1997) [hep-ph/9705234].

[12] V. Ahrens, A. Ferroglia, M. Neubert, B. D. Pecjak and L. L. Yang, JHEP 1009, 097 (2010) [arXiv:1003.5827 [hep-ph]].

[13] A. Ferroglia, B. D. Pecjak and L. L. Yang, Phys. Rev. D 86, 034010 (2012) [arXiv:1205.3662 [hep-ph]]. 
[14] A. Ferroglia, M. Neubert, B. D. Pecjak and L. L. Yang, Phys. Rev. Lett. 103, 201601 (2009) [arXiv:0907.4791 [hep-ph]].

[15] A. Ferroglia, M. Neubert, B. D. Pecjak and L. L. Yang, JHEP 0911, 062 (2009) [arXiv:0908.3676 [hep-ph]].

[16] A. Ferroglia, B. D. Pecjak and L. L. Yang, JHEP 1309, 032 (2013) [arXiv:1306.1537 [hep-ph]].

[17] A. Ferroglia, B. D. Pecjak, L. L. Yang, B. D. Pecjak and L. L. Yang, JHEP 1210, 180 (2012) [arXiv:1207.4798 [hep-ph]].

[18] G. Wang, X. Xu, L. L. Yang and H. X. Zhu, JHEP 1806, 013 (2018) [arXiv:1804.05218 [hep-ph]].

[19] S. Catani and M. H. Seymour, Phys. Lett. B 378, 287 (1996) [hep-ph/9602277].

[20] S. Catani and M. H. Seymour, Nucl. Phys. B 485, 291 (1997) Erratum: [Nucl. Phys. B 510, 503 (1998)] [hep-ph/9605323].

[21] A. Broggio, A. Ferroglia, B. D. Pecjak, A. Signer and L. L. Yang, JHEP 1603, 124 (2016) [arXiv:1510.01914 [hep-ph]].

[22] A. Broggio, A. Ferroglia, B. D. Pecjak and L. L. Yang, JHEP 1702, 126 (2017) [arXiv:1611.00049 [hep-ph]].

[23] K. G. Chetyrkin and F. V. Tkachov, Nucl. Phys. B 192, 159 (1981).

[24] F. V. Tkachov, Phys. Lett. 100B, 65 (1981).

[25] A. V. Kotikov, Phys. Lett. B 254, 158 (1991).

[26] T. Gehrmann and E. Remiddi, Nucl. Phys. B 580, 485 (2000) [hep-ph/9912329].

[27] J. M. Henn, Phys. Rev. Lett. 110, 251601 (2013) [arXiv:1304.1806 [hep-th]].

[28] A. B. Goncharov, Math. Res. Lett. 5, 497 (1998) [arXiv:1105.2076 [math.AG]].

[29] T. Becher and M. Neubert, Phys. Rev. D 79, 125004 (2009) Erratum: [Phys. Rev. D 80, 109901 (2009)] [arXiv:0904.1021 [hep-ph]].

[30] A. V. Belitsky, Phys. Lett. B 442, 307 (1998) [hep-ph/9808389].

[31] M. Czakon and P. Fiedler, Nucl. Phys. B 879, 236 (2014) [arXiv:1311.2541 [hep-ph]].

[32] R. D. Ball et al. [NNPDF Collaboration], JHEP 1504 (2015) 040 [arXiv:1410.8849 [hep-ph]]. 